\section{Defining criteria to choose appropriate destination hospital for trauma patients: Piacenza Local Health Authority's Piacenza trauma algorithm protocol}

\author{
Fabio Mozzarelli, ${ }^{1}$ Stefano Nani, ${ }^{1}$ \\ Enrica Rossi, ${ }^{1}$ Mario Pizzamiglio ${ }^{2}$
}

1 Ambulance Emergency Service, Piacenza Local Health Authority, Piacenza;

2Emergency Department, Guglielmo Da

Saliceto Hospital, Piacenza, Italy

\begin{abstract}
Ambulance crew's choosing of appropriate destination hospital for trauma patients can affect survival and morbidity outcomes. Aim of the present study is to devise a decision-making algorithm in order to allow the best choice of destination hospital for trauma patients and to apply it on an electronic device able to facilitate the decision made by ambulance staff. The method used was analysis of literature data, context and workload with a retrospective observational study. A comparison between the destination hospitals actually chosen and those that could have been chosen with the Piacenza trauma algorithm has been applied. The data shows a 9.5\% ( $\mathrm{P}>0.10)$ more advantageous change in appropriateness in the choice of medical facility and a $1.4 \%$ increase in admissions to the Emergency Department of the provincial hospital. The creation and use of a medical protocol and its consequent installation on an electronic device (tablet) that can be shared over a computer platform could help medical staff make appropriate pre-hospital choices as regards the destination hospital for trauma patients.
\end{abstract}

\section{Introduction}

The prehospital organization and management of trauma patients represents a very important challenge for emergency medical services. In the Western world, trauma injury represents the first cause of death and invalidity in subjects up to 44 years of age and the third cause amongst the general population. ${ }^{1}$ Starting in the 1990s, a number of intra- and extra-hospital systems known as trauma systems was introduced in the United States, Canada and various European countries to improve trauma patient care. These organizational-management systems are based on trau- ma centers, highly-specialised hub centers serving large catchment areas. ${ }^{2}$ The epidemiological data available for Italy indicate that the establishment of a trauma center requires a workload of 450 severe traumas per year per million inhabitants and no less than 250 cases per year are required to maintain the skill sets of the medical teams. ${ }^{3}$ This introduces the issue of avoidable death, i.e. those trauma patients who would have had greater chances of survival if they had been assessed and treated correctly and, above all, sent to the most appropriate hospital facility. In line with the findings of the American College Surgeons Committee on Trauma, certain research studies conducted in Italy have calculated that the percentage of potentially avoidable deaths to be somewhere between 32 and $40 \%$ and the percentage of certainly avoidable deaths has been estimated at between 11 and 18\%.,5 Correct identification of the severity of the patient's condition, consequent medical treatment and the most advantageous choice of hospital given the injuries sustained are, in addition to transfer times, the main variables impacting the survival and morbidity of trauma patients. The emergency medical system must abide by the golden hour concept and the THREE Rs - get the right patient to the right hospital in the right time - are particularly apt.

A number of articles were identified during the literature search, including one French paper highlight the importance of transferring severe trauma patients, identified using specially-devised triage schemes, straight to the hub trauma center. ${ }^{6}$ Some authors of scientific publications specified that in accidents occurring in rural settings patients are taken to trauma centers in between 5 and $10 \%$ of cases, and that the greatest proportion of deaths for severe trauma occur in decentralized emergency departments. ${ }^{7,8}$ Some researchers claim that anatomical and physiological criteria can be used to identify with greater appropriateness those patients with severe injuries, in order to allow a more adequate use of the resources available. ${ }^{9,10}$ One interesting article on the situation in Italy focused on the centralization of major trauma by analysing the injury mechanism. The research was conducted at the Emergency Department of Florence's Ospedale Careggi and reported that many of the patients defined as having major trauma according to physiological or anatomic criteria presented a higher degree of criticality than those referred according to the event dynamics mechanism alone (odds ratio $30.35,95 \%$ confidence interval 21.09-43.65, $\mathrm{P}<0.0001) .^{11}$ Conversely, one research article that conflicts with those mentioned so far, suggests not underestimating the dynamics criterion, especially for falls from a height ${ }^{12}$ and one analysis conducted in Australia, as suggested by the American College of Surgeons Committee on
Correspondence: Fabio Mozzarelli, Emergency Department, Piacenza Hospital, via Antonio Anguissola 15, Piacenza, Italy.

Tel/Fax: +39.0523 .301111$

E-mail: fabio.mozzarelli@gmail.com

Key words: Algorithm; Trauma; Piacenza; Triage; Hospitalization

Contributions: FM took care of data processing and drafting of the article; SN collected data; MP and ER critically evaluated the process.

Conflict of interest: the authors declare no potential conflict of interest.

Received for publication: 9 June 2015 .

Revision received: 26 October 2015.

Accepted for publication: 26 October 2015 .

This work is licensed under a Creative Commons Attribution 4.0 License (by-nc 4.0).

(C) Copyright F. Mozzarelli et al., 2016

Licensee PAGEPress, Italy

Emergency Care Journal 2016; 12:5359

doi:10.4081/ecj.2016.5359

Trauma, revealed that in road accidents the longer time required to remove patients from the vehicle is statistically significant for severe injury. ${ }^{13}$ Studies have also been conducted on the difference between rapid transfer of polytrauma patients directly to trauma centers and transfer to medical facilities with less-specialized resources that are closer to the event. The authors point out that subjects who were sent to less-specialized hospitals had higher mortality rates and that their involvement in the trauma system network can be detrimental. ${ }^{14}$

The aims of this paper are to analyze the pathways for the appropriate hospitalization of trauma patients and to devise a complexity-oriented decision-making algorithm. Furhter, we aim to compare the devised protocol with the information present in scientific literature. A final goal is to implement the decision-making algorithm using a telemedicine system that can help medical staff choose the most suitable facility given the criticality of the patient's condition and the resources available.

\section{Materials and Methods}

Figure 1 summarizes the approach chosen to allocate injured patients within the local area to the hospital facility best suited to the injuries sustained. The medical protocol was developed using epidemiological data and workload data for recent years considering the orographic and demographic characteristics of the province of Piacenza, the local and inter- 
provincial hospitals available and the approaches used in literature such as those proposed by advanced trauma life support, ${ }^{15}$ prehospital trauma life support, ${ }^{16}$ Piacenza Local Health Authority prehospital triage, ${ }^{17}$ trauma team activation by Ulleval University Hospital, ${ }^{18}$ trauma triage criteria, ${ }^{9}$ Centers for Disease Control of Atlanta, ${ }^{19}$ and the Ferrara ambulance service centralization criteria. ${ }^{20}$

This instrument also considers certain hospitals outside the province, as according to the hub and spoke system, Major Hospital of Parma is the hub Trauma Center also for Piacenza and Reggio Emilia, whereas Major Hospital of Cremona is the first level trauma center neighbouring the province of Piacenza, in an area that has always been devoid of its own medical facilities.

In the Piacenza trauma algorithm flow chart, the choice of hospital follows the approach of triage based on a series of indices to be measured and/or observed. Each assessment set is allocated one or more hospitals. Therefore, if a patient criterion or characteristic is observed for the first item it is not necessary to proceed further as the chart suggests the destination hospital. Conversely, if no index coincides with the injured patient being observed, the user moves on to the next assessment block.

The first set dedicated to physiological criteria includes the alteration of certain values such as a Glasgow coma scale (GCS) score of less than 9 points, severe hypotension, significant respiratory rate alteration and burns involving the airways. The above indices can be used both individually and jointly; however, the alteration of just one is sufficient to direct the patient to the hub trauma center, which, in the case of the province of Piacenza, is Major Hospital of Parma and the transfer is usually by helicopter. When the aircraft cannot be used, injured patients are transferred to the provincial trauma center.

The second item listing anatomical injuries refers to open injuries of the head, neck, chest and limbs, crush injuries, skin-stripping injuries and limb mutilation or amputation. It also includes suspected fracture of two or more long bones or a hip, neuromotor deficits or sensory alterations (with a GCS score of between 9 and 13) and, last but not least, and burns covering more than $20 \%$ of the body's surface area. Again, just one criterion is sufficient to direct the injured patient to the hub trauma center or, if this is not possible, to the provincial facility.

The third slot contains two special situations - patients who are more than 20 weeks pregnant and children under 10 years of age in which, given the special condition of the subjects and as gynaecology and paediatric specialists are only present at Piacenza Hospital, the patient must be taken to this facility (or Cremona Hospital if closer).
The last section lists the event mechanisms characterized by a high-energy exchange between the individual and the injuring agent. The list includes falls from a height or, for road accidents, crushing of the vehicle, death of other passengers in the crash and suspicion of vehicles travelling at high speed.

In the case of pedestrians and motorcyclists or cyclists it is necessary to look for clues suggesting high-energy collisions. In the absence of other anatomical/physiological evidence, the major dynamics mechanisms described suggest the patient should be transferred to local first aid services or the closest hospital, as recent evidence published in international guidelines has reduced the emphasis placed on this kind of criterion. ${ }^{21}$ However, if effica- cious airway management is not obtained at the local facility or if the injured patient presents severe haemodynamic instability, the individual can be taken to the closest hospital as all of Piacenza's hospital facilities have a life support specialist on duty around the clock.

The fleet of vehicles used by Piacenza Local Health Authority's emergency service is fitted with the Ortivus MobiMed Smart ${ }^{\mathrm{TM}}$ telemedicine system (Ortivus, Danderyd, Sweden), which allows data transmission and sharing over a dedicated virtual network (Figure 2). This appliance makes it possible to exchange information, clinical data and vital parameters whilst also authorizing the management of clinical documentation such as the drafting of the patient's medical records, the consultation

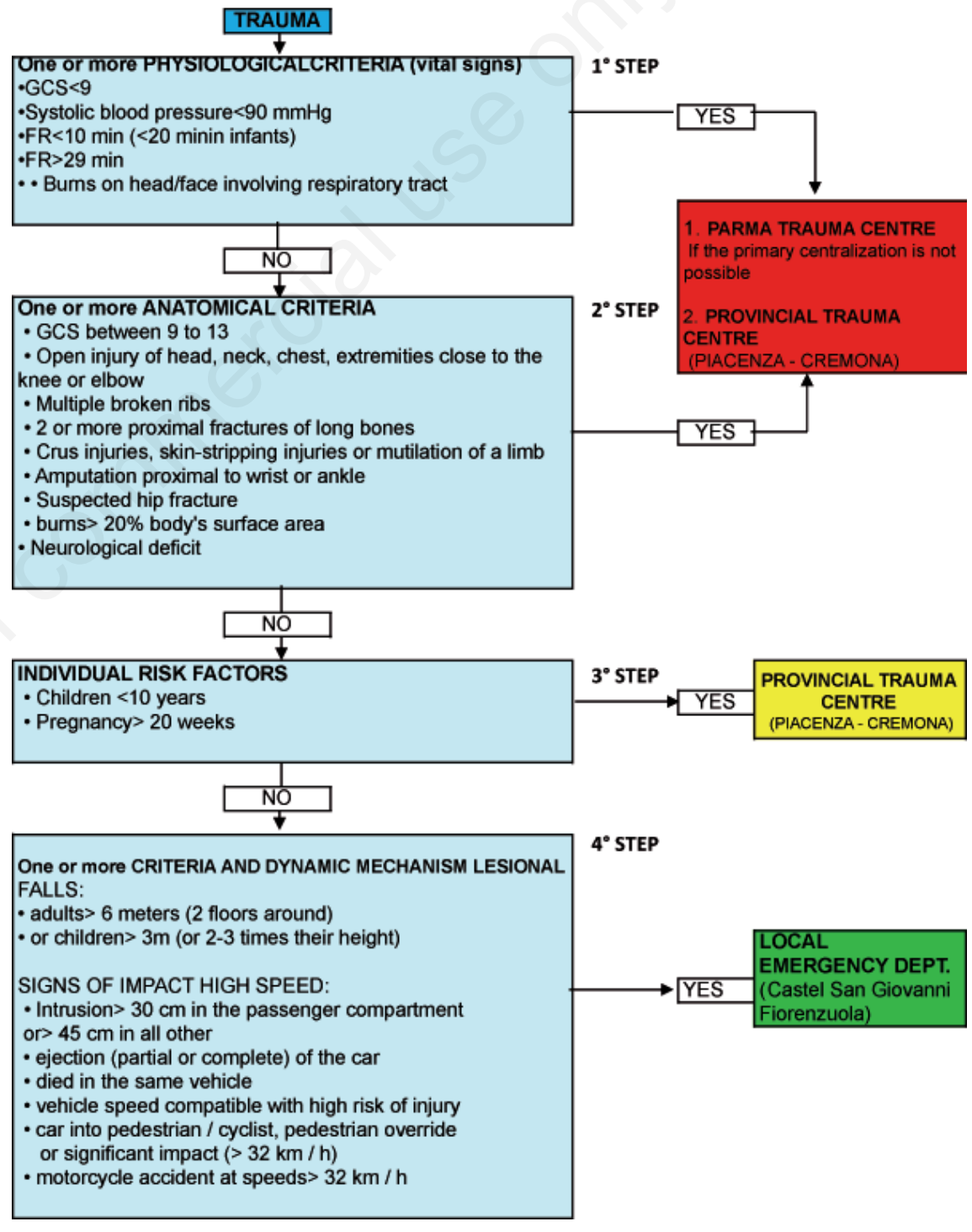

Figure 1. Method used to allocate injured patients within the local area to the hospital facility best suited to the injuries sustained. 
and filling out of certain assessment schemes, scores and scales and also allows the user to consult clinical protocols, diagnostic, therapeutic and care pathways and guidelines. The instrument also contains algorithms that facilitate decision-making concerning case severity and complexity. The Piacenza trauma algorithm was installed on the device in checklist mode to offer local emergency nursing crews the possibility of consulting it as an aid to decision-making as to the most suitable medical facility, limiting the degree of subjectivity of the individual operator and guaranteeing homogeneity in care behaviour.

The appropriateness of injured patient allocation was explored in a retrospective study to evaluate how patients would have been distributed in 2013 had the Piacenza trauma algorithm been used. Patients transferred to medical facilities outside the province, with the exception of Major Hospital of Parma and Major Hospital of Cremona, were excluded from the analysis. All trauma injury patients treated by professional ambulance crews and subsequently transferred to the various emergency services were included. For greater consistency, the study only involved patients who were injured in municipalities belonging to the province in which access to the emergency Department could involve both a local hospital and the provincial trauma center in Piacenza (Cremona or Parma), and therefore excluded patients injured in urban, suburban and even extra urban areas who, regardless of the severity of the event, would only have been taken to Piacenza Hospital, as it was both the closest and the best equipped facility.

To improve the analysis, a random caseload was created from which a sample of 345 units was obtained. The selected patients were evaluated using the patient record filled out by the local emergency system staff for each patient, the computerized record used by the ambulance call center when receiving and managing callouts, engineering 118@NET the regional computer platform, Dedalus healthcare systems group the electronic record used in emergency services, the injury severity score (ISS), measured using the information collected from the emergency record for critical patients (yellow and red codes) alone and only for injured patients transferred to hospitals in the Piacenza area.

By studying these instruments and comparing them with the Piacenza trauma algorithm we obtained the information needed to compare the actual destination of trauma patients with that which could have been chosen in 2013. The sample size was calculated using Raosoft software, by setting a power value of $90 \%$, a confidence interval of $95 \%$ and a prevalence index of $50 \%$. Randomization was performed using a table created with the random number generation system provided on the
Emilia-Romagna Regional Authority website. The data was collected and processed using a Microsoft ${ }^{\circledR}$ Office Excel 2007 spreadsheet. The $\chi^{2}$ test was used for statistical comparisons. Differences were considered statistically significant when $\mathrm{P}>0.05$.

\section{Results}

The analysis of the data collected in the observational study showed that $9.5 \%$ of patients $(\mathrm{P}>0.10)$ would have been sent to a different destination hospital than that which was actually chosen. Of injured patients classified as having low criticality, $5.4 \%$ were transferred to the hub trauma center in Piacenza, despite the fact that they could have been taken to a local emergency Department. Of injured patients identified as having an intermediate complexity, $3.8 \%$ were taken to peripheral emergency services but should have been taken to the hub trauma center and $0.3 \%$ of high severity patients who should have been taken to the city hospital or, better still, to the Parma trauma center were taken to a local hospital (Figure 3). Another conclusion that can be drawn concerns the impact of patient distribution to the various emergency services had the criteria of the new algorithm been adopted. By applying the appropriate proportions there would have been a $1.4 \%$ increase in admissions to Piacenza Emergency Department (Figure 4).

\section{Discussion}

The aim of the Piacenza trauma algorithm and its installation on Ortivus MobiMed Smart ${ }^{\mathrm{TM}}$ was to direct the professional to a reasoned, homogeneous and appropriate choice of destination hospital in trauma patients in the province of Piacenza. The corresponding flow chart was devised taking into account the orographic and demographic characteristics of the province of Piacenza, considering the arrangement of its hospital facilities and those of the greater Western Emilia area, as well as the regional organization of the trauma system, on the basis of papers published in scientific literature, the best and most up-to-date scientific evidence and information concerning the workload and analysis of the data obtained from the retrospective study.

The limitations of this study could be the decision to adopt an algorithm characterized by triage criteria, which may lead to over-or underestimation. The increase in patients directed to Piacenza Emergency Department was estimated in quantitative terms (1.4\%); however, the caregiving weight and clinical commitment that these new patients would generate was not studied.

Patients with low criticality injuries represented over $90 \%$ of total caseload; in the light of this data new organizational measures should be taken to dedicate greater attention to this type of patient in emergency departments. Literature has consistently shown that

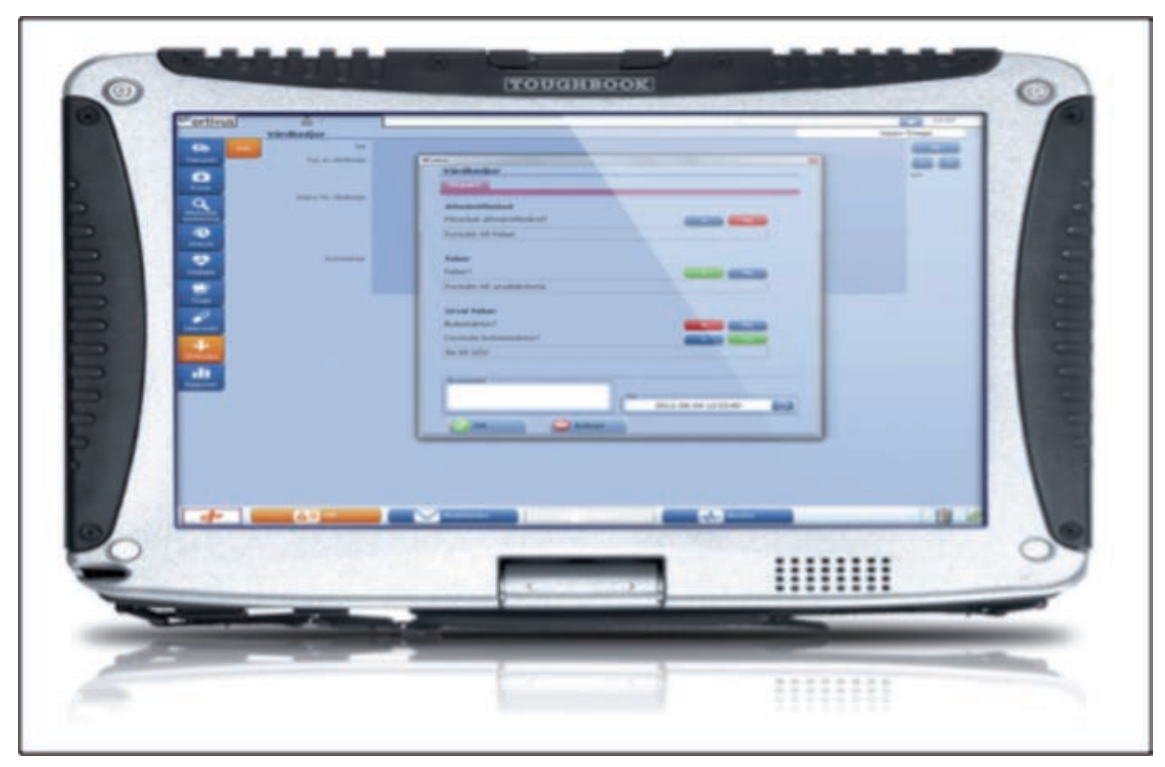

Figure 2. Ortivus MobiMed Smart ${ }^{\mathrm{TM}}$. 


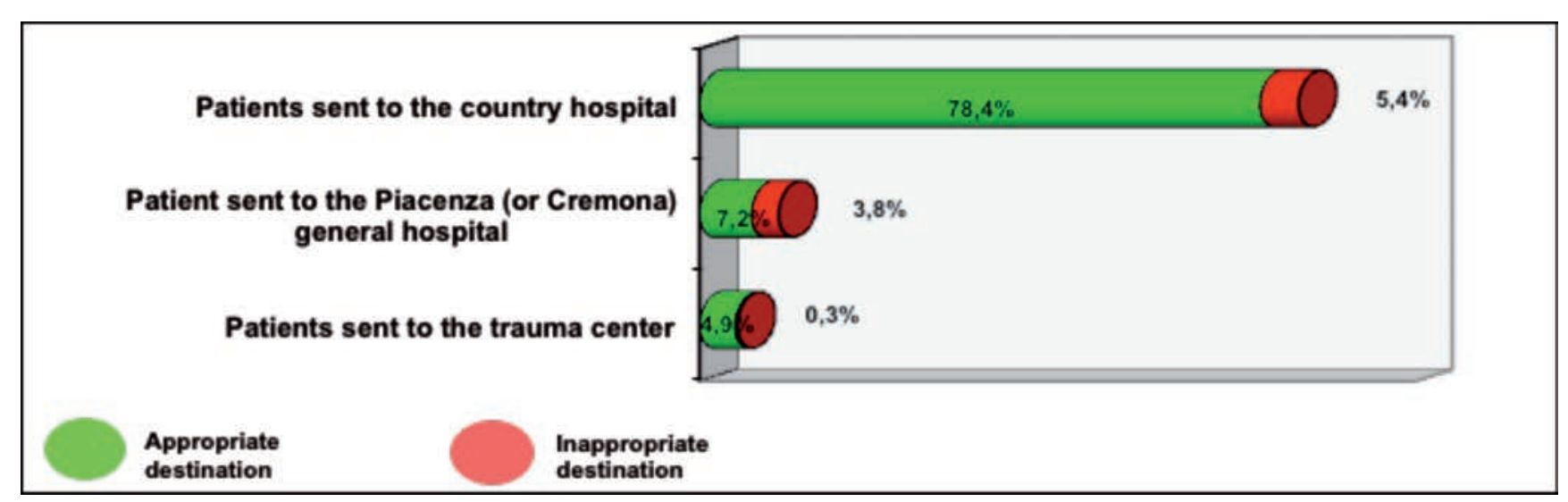

Figure 3. Distribution of the injured patients in Piacenza district, 2013.

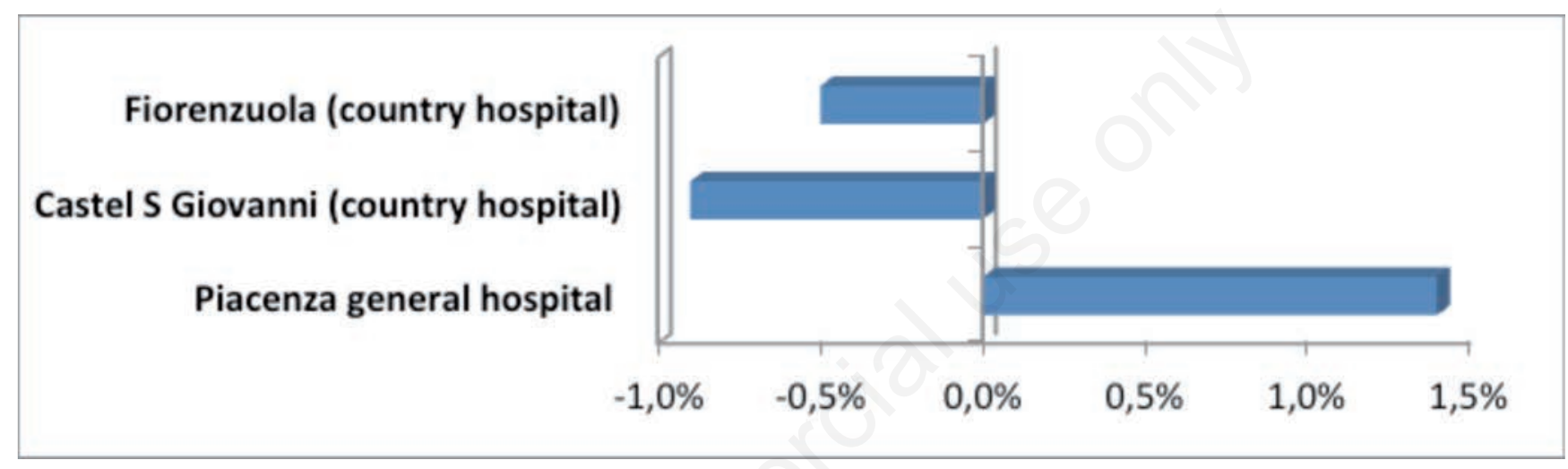

Figure 4. Estimated increase access to the emergency department in Piacenza.

the choice of hospital facility can impact survival indices and invalidating outcomes. Use of the Piacenza trauma algorithm could provide professionals with valid support when choosing destination hospitals. The observational research showed that certain choices concerning the destination hospital did not comply with the criteria suggested by the algorithm. Despite not being statistically significant, this portion, which is equal to $9.5 \%$, can be improved. With a view to the future development of the pre-hospital management of trauma patients, the conduct of a observational (cohort, case-crossover, before/after) study could provide an opportunity to further investigate the validity of the Piacenza trauma algorithm, to ascertain with more suitable instruments the care workload and clinical complexity of the majority of patients who would be admitted to Piacenza Emergency Department.

\section{Conclusions}

The creation of the Piacenza trauma algorithm and its application on the Ortivus MobiMed Smart ${ }^{\mathrm{TM}}$ telemedicine system will make it possible to choose the destination hospital for trauma patients provided by the regional and provincial hospital network with greater accuracy and uniformity. Moreover, the device stores the decision-making steps selected by the professional for potential future verification and analysis.

\section{References}

1. Chiara 0, Cimbanassi S, Fava A, Vesconi S. La rete organizzativa per la gestione del trauma in Italia. Emerg Care J 2005;1:36-42.

2. Mann NC, Mullins RJ, MacKenzie EJ, et al. Systematic review of published evidence regarding trauma system effectiveness. J Trauma 1999;47:25-33.

3. Chiara 0, Cimbanassi S. Organized trauma care: does volume matter and do trauma centers save lives? Curr Opin Crit Care
2003;9:510-4.

4. Chiara 0, Scott JD, Cimbanassi S, et al. Trauma deaths in an Italian urban area: an audit of pre-hospital and in-hospital trauma care. Injury 2002;33:553-62.

5. Dinh MM, Bein K, Roncal S, et al. Redefining the golden hour for severe head injury in an urban setting: the effect of prehospital arrival times on patient outcomes. Injury 2013;44:606-10.

6. Tazarote K, Cesaréo E, Sapir D, et al. Update on prehospital emergency care of severe trauma patients. Ann Fr Anesth 2013;32:477-82.

7. McSwain N, Rotondo M, Meade P, et al. A model for rural trauma care. Brit J Surg 2012;99:309-14.

8. Gomez D, Berube M, Xiong W, et al. Identifying targets for potential interventions to reduce rural trauma deaths. A population-based analysis. Trauma 2010;69:633-9.

9. Cook CH, Muscarella P, Praba AC, et al. Reducing overtriage without compromising outcomes in trauma patients. Arch 
Surg-Chicago 2001;136:752-6.

10. Kohn MA, Hammel JM, Bretz SW, et al. Trauma team activation criteria as predictors of patient disposition from the emergency department. Acad Emerg Med 2004;11:1-9.

11. Bambi S, Ruggeri M, Rossi S, et al. La centralizzazione del trauma maggiore per criterio di meccanismo lesionale: analisi biennale della casistica in un dipartimento di emergenza toscano di $2^{\circ}$ livello. Scenario 2009;26:6-14.

12. Velmahos GC, Jindal A, Chan LS, et al. "Insignificant" mechanism of injury: not to be taken lightly. J Am Coll Surg 2001;192:147-52.

13. Palanca S, Taylor DM, Bailey M, Cameron PA. Mechanisms of motor vehicle accidents that predict major injury. Emerg Med Australas 2003;15:423-8.

14. Nirula R, Maier R, Moore E, et al. Scoop and run to the trauma center or stay and play at the local hospital: hospital transfer's effect on mortality. J Trauma 2010;69:595-9.

15. American College of Surgeons Committee on Trauma. Advanced trauma life support. 9th ed. Chicago, IL: American College of Surgeons ed.; 2012.

16. American College of Surgeons Committee on Trauma. Prehospital trauma life support. 5th ed. Turin, Italy: Centro Scientifico Editori; 2003.

17. Arvedi M, Bologna G, Cammarata S, et al. Processi di centralizzazione in: il politrauma nell'Azienda USL Piacenza 2005. Available from: http://www.ausl.pc.it/pubblicazioni_aziendali/atti_convegni/trauma_2005/docs/IL\%20POLITRAUMA.pdf

18. Rehn M, Eken T, Kruger AJ, et al. Precision of field triage in patients brought to a trauma centre after introducing trauma team activation guidelines. Scand J Trauma Resusc Emerg Med 2009;17:1.

19. Center for Disease Control and Prevention. Guidelines for the field triage of injured patients. Recommendations of the National Expert Panel on Field Triage 2011. Available from: http://www.cdc .gov/mmwr/preview/mmwrhtml/rr6101a1.h tm

20. Melcarne L, Ricciardelli A, Melandri R, et al. La centralizzazione del politrauma, studio della realtà ferrarese e simulazione della presenza di un protocollo concordato. Emerg Care J 2011;3:15-9.

21. Center for Disease Control and Prevention. Guidelines for field triage of injured patients. Recommendations of the National Expert Panel on Field Triage 2009. Available from: www.cdc.gov/mmwr/ PDF/rr/rr5801.pdf 